Broman-Fulks, J. J., Green, B. A., Berman, Olatunji, B. O., M. E., Arnau, R. C., Deacon, B. J., \& Sawchuk, C. N. (2008). The latent structure of anxiety sensitivity - revisited. Assessment, 15(2): 188-203. (June 2008) Published by Sage (ISSN: 1552-3489). doi:10.1177/1073191107311284

\title{
The Latent Structure of Anxiety Sensitivity-Revisited
}

Joshua J. Broman-Fulks, Bradley A. Green, Mitchell E. Berman, Bunmi O. Olatunji, Randolph C. Arnau, Brett J. Deacon, and Craig N. Sawchuk

\begin{abstract}
Anxiety sensitivity has been implicated as a risk factor for the development and maintenance of panic and other anxiety disorders. Although researchers have generally assumed that anxiety sensitivity is a dimensional, rather than categorical, variable, recent taxometric research has raised questions concerning the accuracy of this assumption. The present study examined the latent structure of anxiety sensitivity by applying four taxometric procedures (MAXEIG, MAXCOV, MAMBAC, and L-Mode) to data collected from two large nonclinical samples $(\mathrm{n}=1,025$ and $\mathrm{n}=744)$ using two distinct measures of anxiety sensitivity (Anxiety Sensitivity Profile and Anxiety Sensitivity Index-Revised). In contrast to previous taxometric analyses of anxiety sensitivity, results of the present research provided convergent evidence for a latent anxiety sensitivity dimension. Several potential explanations for the discrepancy between these findings and those of previous research are discussed, as well as the implications of these findings for the conceptualization and measurement of anxiety sensitivity.
\end{abstract}


Anxiety sensitivity refers to the fear of anxiety-related sensations associated with beliefs that these sensations can have harmful consequences (Reiss \& McNally, 1985). Accumulating evidence appears to indicate that anxiety sensitivity is a cognitive vulnerability that predisposes individuals to panic attacks and other anxiety pathology (Ehlers, 1995; Maller \& Reiss, 1992; McNally, 2002; Schmidt, Lerew, \& Jackson, 1997; Schmidt, Zvolensky, \& Maner, 2006). Although no explicit statement was initially made regarding the latent structure of anxiety sensitivity, it appears that anxiety sensitivity was initially conceptualized as a dimensional variable, present to a greater or lesser extent in all individuals (Reiss \& McNally, 1985). Consistent with this theoretical formulation, the vast majority of the anxiety sensitivity literature has developed on the assumption that anxiety sensitivity is dimensional (continuous) rather than categorical at the latent level.

Evidence of the dimensionality assumption can be found throughout the anxiety sensitivity literature. Measures of anxiety sensitivity have generally been developed for the purpose of locating a point on the continuum of the fear of anxiety and anxiety-related sensations where a particular individual falls (e.g., Anxiety Sensitivity Index [ASI]; Peterson \& Reiss, 1992; Anxiety Sensitivity Profile [ASP]; Taylor \& Cox, 1998a), rather than determining whether the individual possesses healthy versus unhealthy levels of anxiety sensitivity. In addition, researchers frequently refer to individuals as possessing various degrees of anxiety sensitivity (i.e., high, moderate, low, etc.; e.g., Broman-Fulks, Berman, Rabian, \& Webster, 2004; Maller \& Reiss, 1992; Watt, Stewart, Birch, \& Bernier, 2006), rather than belonging to an anxiety sensitivity category (i.e., normal vs. pathological). However, the lack of an explicit statement regarding the latent structure of anxiety sensitivity appears to have resulted in some confusion among anxiety sensitivity researchers regarding the most appropriate methodological approaches to take when studying anxiety-related fears.

Inconsistent with the dimensional conceptualization of anxiety sensitivity, for example, some investigators have elected to dichotomize anxiety sensitivity by means of arbitrary cutoff scores for analytical, selection, or convenience purposes, thereby treating anxiety sensitivity as a categorical variable, at least at the manifest level of measurement (e.g., Asmundson, Norton,Wilson, \& Sandler, 1994; Broman-Fulks et al., 2004; Donnell \& McNally, 1990; Harrington, Telch, Abplanalp, \& Hamilton, 1995). Although dichotomization of a continuous variable may be desirable or necessary because of methodological or practical reasons, dichotomization of a continuous variable can 
also have several undesirable effects, including decreased statistical power and a loss of potentially important information (Cohen, 1983). If anxiety sensitivity is truly dimensional, research designs that consider the entire range of anxiety sensitivity would maximize statistical power and minimize information loss. However, if anxiety sensitivity is categorical, then research should attempt to identify the indicators that best discriminate between individuals affected by anxiety sensitivity and those who are not. Thus, knowing the latent structure of anxiety sensitivity is of utmost importance because the conceptualization, measurement, and approach to studying anxiety sensitivity are fundamentally informed by its latent structure. Fortunately, the latent structure of anxiety sensitivity is an empirical question, one for which appropriate research and statistical techniques are available to derive an answer.

Taxometrics refers to a set of statistical procedures designed to discern the latent structure (categorical vs. continuous distribution) of phenomena using multiple fallible indicators of the construct of interest (Meehl \& Golden, 1982). Taxometric methodology does not rely on traditional statistical significance testing, instead requiring that several independent procedures (i.e., consistency tests) provide convergent evidence either in support or against the existence of a taxon. The utility of taxometric procedures has been clearly established by its ability to correctly identify real world phenomena known to be taxonic, such as biological sex (Meehl \& Golden, 1982), and researchers have begun to apply taxometric procedures to the study of various psychological constructs, including depression (e.g., Ruscio \& Ruscio, 2000), schizotypy (Lenzenweger \& Korfine, 1992), and posttraumatic stress disorder (e.g., Broman-Fulks et al., 2006; Ruscio, Ruscio, \& Keane, 2002). With regard to anxiety sensitivity, a number of taxometric investigations have recently been published. With one exception, the results of these taxometric analyses have suggested that anxiety sensitivity may in fact be a categorical variable.

The first attempt to examine the latent structure of anxiety sensitivity via the taxometric method was made by Taylor, Cox, Freeman, McNally, Stewart, and Swinson (as reported in Taylor, Rabian, \& Federoff, 1999). Maximum covariance (MAXCOV) analyses were conducted using 8 of the 16 items on the ASI. Indicators of the conjectured anxiety sensitivity taxon were generated by combining the $8 \mathrm{ASI}$ items into four pairs in an attempt to increase the potential for covariance among the items and obtain more reliable results than would be achieved using the individual items as indicators. Of six MAXCOV plots 
generated, three appeared to indicate that anxiety sensitivity is nontaxonic, whereas the remaining three were ambiguous. Based on these results, the authors suggested that anxiety sensitivity is most accurately conceptualized as representing a latent dimension. However, several critical methodological limitations raised concerns regarding the interpretation of these findings. Specifically, the indicators derived from the ASI represented various amalgamations of ASI items that were not empirically based, and the psychometric characteristics of the indicators, which could have a substantial impact on the ability of these taxometric procedures to detect a taxon if one existed, were inadequately described. Furthermore, although consistency tests are considered a necessary component of taxometric procedures, consistency testing was not reported despite ambiguous results.

In a subsequent study, Schmidt, Kotov, Lerew, Joiner, and lalongo (2005) investigated the latent structure of a cognitive vulnerability for panic (similar to anxiety sensitivity) in 1,296 military cadets. MAXCOV and MAXEIG (Maximum eigenvalue) analyses were performed using indicators derived from the total scores of two anxiety sensitivity measures: the ASI and Body Sensations Questionnaire (Chambless, Caputo, Bright, \& Gallagher, 1984), and a measure of body vigilance (the Body Vigilance Questionnaire; Schmidt, Lerew, \& Trakowski, 1997).

Results of the MAXCOV and MAXEIG analyses were interpreted as providing support for a latent anxiety sensitivity taxon, with an estimated taxon base rate of $18 \%$.

However, again, several notable limitations were apparent. For example, as noted above, taxometric procedures are dependent on multiple independent procedures converging on either taxonic or nontaxonic findings. However, the Schmidt et al. study employed a relatively weak set of consistency tests, including the use of two highly redundant taxometric procedures (MAXCOV and MAXEIG) and the interpretation of a very limited number of plots (only three MAXCOV and three MAXEIG plots). The authors reported that mean above minus below a cut (MAMBAC) analyses were also conducted (MAMBAC represents a mathematically independent taxometric procedure), though the MAMBAC apparently failed to produce consistently taxonic-shaped plots. The authors attributed MAMBAC's inability to generate taxonic findings as being a result of the "relatively low base rate" of the taxon and nuisance correlations among the indicators (Schmidt et al., 2005).

In an attempt to address some of these limitations and more specifically investigate the latent structure of anxiety sensitivity, Bernstein and colleagues have conducted a 
series of taxometric analyses using relatively diverse samples. For example, Bernstein et al. (2006) used MAXCOV and MAMBAC to examine the latent structure of anxiety sensitivity in five samples of college students from six different countries (173 U.S. college students were combined with 478 Canadian students to create a North American sample). Four composite indicators were created by summing the items on each of the four subscales of the revised version of the ASI (Anxiety Sensitivity Index-Revised [ASI-R]; Taylor \& Cox, 1998b). Visual inspection of the resulting plots suggested evidence of taxonic structure in each of the five samples. MAXCOV base rate estimates ranged between $12 \%$ and $20 \%$, which were similar to the estimates reported by Schmidt et al. (2005). In a second study Bernstein, Zvolensky, Weems, Stickle, and Leen-Feldner (2005) tested the taxonic structure of anxiety sensitivity in a sample of nonclinical youth, again using the MAXCOV and MAMBAC procedures. Multiple combinations of Childhood Anxiety Sensitivity Index (CASI; Silverman, Fleisig, Rabian, \& Peterson, 1991) items (i.e., some items were used more than once) were used to create manifest indicators. Results appeared generally consistent with a latent taxon, with comparable base rates $(M=13.6 \%)$ to previous reports. Bernstein and colleagues (2006) followed up this study with a second analysis of anxiety sensitivity among nonclinical Canadian youth, this time comparing latent structure across male and female subgroups separately. Results of a "short-scale" MAXEIG procedure (every possible pair of nine CASI items served as an output indicator once, with the remaining seven items being summed to form a composite input indicator) and MAMBAC (analyzing each possible combination of nine individual CASI items) analyses were again interpreted as suggestive of a latent taxon, with females showing higher base rate estimates (12\%) than males (7\%). Most recently, Bernstein and colleagues (2007) investigated the latent structure of anxiety sensitivity in a large, nonclinical sample of North American college students. Consistent with their previous reports, results of MAXCOV and MAXEIG analyses appeared to support the existence of an anxiety sensitivity taxon with a mean base rate of .11. Thus, Bernstein and colleagues have been able to replicate their taxonic findings for anxiety sensitivity across several samples. To the best of our knowledge, however, no other independent research groups have published peer-reviewed reports directly examining the latent structure of anxiety sensitivity. Thus, one of the purposes of this study was to replicate the findings of Bernstein and colleagues to improve confidence in the generalizability of their findings. 
In reviewing the extant literature on taxometric studies of anxiety sensitivity, it was also noted that the vast majority of analyses utilized indicators generated from either the ASI or derivations of the ASI (i.e., ASI-R or CASI). However, some researchers have recently raised concerns over the ASI and ASI-R (Olatunji et al., 2005), suggesting that the ASI contains several items that are ambiguously worded and has too few items to adequately measure the full range of the subcomponents of anxiety sensitivity. Furthermore, both the ASI and ASI-R are composed of a mixture of items that assess both cognition (i.e., beliefs that anxiety-related sensations are dangerous) and emotion (i.e., fear of sensations), making it difficult to determine the extent to which anxiety sensitivity is made up of fears, beliefs, or fears based on beliefs (Deacon, Abramowitz, Woods, \& Tolin, 2003). Thus, the present study also sought to replicate prior taxonic findings for anxiety sensitivity using an alternative measure of anxiety sensitivity. We attempted to accomplish these tasks using four taxometric procedures: MAXEIG, MAXCOV, MAMBAC, and L-Mode factor analyses. Based on the predominately taxonic findings of previous taxometric analyses of anxiety sensitivity, it was hypothesized that multiple taxometric procedures would yield a taxonic solution, with a base rate of approximately $10 \%$ to $20 \%$.

\section{GENERAL METHOD}

\section{Consistency Tests}

Taxometric procedures do not rely on traditional statistical significance testing. Rather, a consistency testing approach is implemented to protect against spurious results (Meehl, 1995a; Waller \& Meehl, 1998). If a latent taxon exists, multiple independent taxometric procedures should produce consistent results in support of a taxon. In accordance with this methodological approach, the present study implemented four mathematically independent taxometric procedures to evaluate the latent structure of anxiety sensitivity: MAXEIG (Waller \& Meehl, 1998), MAXCOV (Meehl \& Yonce, 1996; Waller \& Meehl, 1998), MAMBAC (see Meehl \& Yonce, 1994), and L-Mode factor analyses (Waller \& Meehl, 1998). If an anxiety sensitivity taxon truly exists, the results of these four taxometric procedures should converge on a taxonic solution.

All MAXEIG, MAXCOV, MAMBAC, and L-Mode analyses were run using $R$ statistical software ( $R$ Development Core Team, 2005) using algorithms obtained from Ruscio (2006). 1 In addition to analyzing the empirical data, 
these programs can simultaneously create simulated taxonic and dimensional data plots based on data with similar distributional characteristics (i.e., skew, kurtosis, $N$, etc.) to the empirical data (Ruscio, Haslam, \& Ruscio, 2006; Ruscio, Ruscio, \& Meron, 2007). The simulations can then be used to aid in the interpretation of the empirical data plots by comparing them to the plots produced by the simulated dimensional and taxonic data. A detailed description of the simulation programs can be found in Ruscio et al. (2007). In the present research, cases were sorted into putative taxon versus non-taxon membership using Bayes' Theorem in MAXEIG and MAXCOV, the grand mean base rate estimate in MAMBAC, and the nearest mode in L-Mode.2 Although some researchers have voiced concerns over the use of sample specific simulations and the programs that generate them (Beach, Amir, \& Bau, 2005), a recent reanalysis of the data that prompted these concerns has instead provided evidence to support the use of comparison data in taxometric research (Ruscio \& Marcus, 2007).

\section{Statistical Procedures}

MAXEIG. MAXEIG (Waller \& Meehl, 1998) is a multivariate generalization of the frequently used MAXCOV procedure (Meehl \& Yonce, 1996). MAXEIG calculates and plots eigenvalues from the covariance of all remaining indicators across successive intervals of each indicator, thus producing one MAXEIG plot per indicator. If a taxon is present, data typically yield plots with peaked curves. In contrast, dimensional data tend to produce relatively flat plots. Recent research has demonstrated that the use of indicators with skewed distributions can be problematic for the traditional MAXCOV procedure in that MAXCOV plots generated by dimensional data can be frequently misinterpreted as plots generated by a low base-rate taxon (Ruscio \& Ruscio, 2002). In an attempt to provide increased protection against mistakenly inferring taxonic structure from MAXCOV curves that are cusped at the upper end, the current research implemented the MAXEIG procedure, which generates a far greater number of response points and may increase the ability to distinguish taxonic and dimensional variables when indicators are skewed (Ruscio, Ruscio, \& Keane, 2004). As an additional protective feature, the MAXEIG Inchworm Consistency Test (ICT; Waller \& Meehl, 1998) was also included, using 50, 75, and 100 windows and an overlap of .90. The Inchworm Consistency Test has been shown to increase the interpretability of MAXEIG plots and to be particularly helpful in elucidating the 
presence of extremely high or low base-rate taxa (see Waller \& Meehl, 1998).

MAXCOV. The MAXCOV (Meehl \& Yonce, 1996) procedure functions by examining the patterns of covariance between two indicators across successive, nonoverlapping intervals of a third indicator. When the resulting function is plotted, taxonic variables will produce a clear peak at the interval that contains the largest mixture of taxon and nontaxon groups (i.e., the hitmax). In contrast, if the variable is nontaxonic, the graphs will remain relatively flat because relatively stable covariances across the distribution of scores. Although MAXCOV output can be difficult to interpret when indicator distributions are skewed (Ruscio \& Ruscio, 2002), the present study included MAXCOV analyses (in addition to three other taxometric procedures) to be consistent with previous anxiety sensitivity taxometric research, which has often relied on MAXCOV as the primary method of analysis.

MAMBAC. The MAMBAC (Meehl \& Yonce, 1994) procedure is based on the assumption that if two discrete groups exist (i.e., taxon and nontaxon), mean differences between groups will occur on valid indicators of group membership. When mean differences on one variable are plotted as a function of another variable, taxonic constructs tend to produce peaked plots at the point that best differentiates the two groups, whereas dimensional constructs produce dish-shaped plots.

L-Mode. L-Mode (Waller \& Meehl, 1998) combines all candidate indicators and conducts an exploratory factor analysis on the covariances between indicators. Factor score estimates for the first principal factor are computed and used to plot a factor-score probability density distribution (Arnau, Thompson, \& Cook, 2001). When the distribution of scores for the first principal factor is examined, taxonic variables produce a bimodal distribution of factor scores, whereas dimensional construct factor scores tend to be unimodally distributed.

\section{Taxometric Graph Ratings}

Interpretation of taxometric results relies heavily on the visual inspection and ratings of various plots. Because of the subjective nature of such analyses, the present study employed four experienced taxometricians to independently examine and rate the data plots as indicative of taxonicity, continuity, or ambiguity. 3 Simulated taxonic and 
dimensional data with similar distributional characteristics to the research data were also provided to the raters.

Raters were encouraged to compare research data plots with the simulations and to use the simulations as an additional source of information in making their decision about the shape of the empirical data plots.

\section{STUDY 1}

\section{Method}

\section{Participants}

Participants for Study 1 were 1,025 undergraduate students from two large universities. The sample contained more females (56.1\%) than males, and ranged in age from 18 to $70(M=20.55, S D=4.44)$. Ethnic breakdown for the sample included $76.8 \%$ White, $11.2 \%$ African American, 3.3\% Hispanic, 5.7\% Asian, 1.7\% Native American, and $1.4 \%$ other. Participants volunteered to complete the study in exchange for required or extra credit for various psychology courses. Students electing to participate in psychological research were able to choose from several studies being conducted in their respective psychology department at that time. Previous anxiety sensitivity taxometric investigations have reported base rate estimates of approximately $16 \%$ to $18 \%$ in college student populations. Thus, the large university sample used in the present study should provide sufficient numbers of taxon members to allow for the detection of a latent anxiety sensitivity taxon if one exists.

\section{Measures}

ASP. The ASP (Taylor \& Cox, 1998a) is a 60-item selfreport questionnaire that assesses beliefs about the harmful consequences of anxiety-related sensations. The ASP was originally designed to assess six domains of anxiety sensitivity, including fears of: (a) cardiovascular symptoms,

(b) respiratory symptoms, (c) gastrointestinal symptoms, (d) publicly observable anxiety reactions, (e) dissociative and neurological symptoms, and (f) cognitive dyscontrol (Taylor \& Cox, 1998a). Although at least one confirmatory factor analysis has supported the six-factor solution (Van der Does, Duijsens, Eurelings-Bontekoe, Verschuur, \& Spinhoven, 2003), evidence from several exploratory factor analytic studies have suggested that the ASP assesses a general anxiety sensitivity factor and only four lower order 
factors: (a) fear of arousal-related symptoms, (b) fear of cognitive dyscontrol and dissociation, (c) fear of gastrointestinal symptoms, and (d) fear of cardiac symptoms (e.g., Olatunji et al., 2005). Construct validity has been established based on the ASP's strong correlations with the original version of the ASI $(r=.62)$. The ASP has demonstrated excellent internal consistency $(\square=.98)$ with all 60 items showing adequate item-total correlations (range $=$ .50-.76; Olatunji et al., 2005).

\section{ASP Indicator Selection}

Composite indicators of the conjectured anxiety sensitivity taxon were created by summing the items on each of ASP subscales. As noted above, the ASP was initially designed to assess six domains of anxiety sensitivity, and this structure has received subsequent support via confirmatory factor analysis (Van der Does et al., 2003).

However, several recent factor analytic studies have suggested that the ASP may be better represented as measuring four lower order factors rather than the six it was initially designed to assess (e.g., Olatunji et al., 2005). Based on these findings, the present study utilized two sets of ASP indicators representing the six- and four-factor solutions, respectively. Summation of ASP subscale items increases the potential range of the indicators, thus yielding more reliable indicators than would be obtained if individual item responses were used as indicators. The validity and nuisance covariance of the ASP- 6 and ASP-4 indicators were examined. As noted above, cases were sorted into putative taxon versus non-taxon membership using Bayes' Theorem in MAXEIG and MAXCOV, the grand mean base rate estimate in MAMBAC, and the nearest mode in L-Mode. Nuisance correlations among the ASP-6 indicators were slightly higher than is typically desirable (average correlation of .44 for the taxon class and .50 for the complement class). However, relatively even distribution of nuisance correlations across the conjectured taxon and complement groups is generally not problematic (Meehl, 1995b; Ruscio et al., 2006). Furthermore, as is desirable, the average correlation among the full sample was significantly higher $(r=.74)$. The nuisance correlations among the ASP-4 indicators were somewhat lower than with the ASP- 6 indicators and were relatively evenly distributed across the taxon $(r=.33)$ and nontaxon $(r=.46)$ groups. The average correlation of the ASP-4 indicators in the full sample was also considerably higher $(r=.70)$.

Indicator validities were estimated by submitting the within-group and total correlation values into a formula 
provided by Meehl and Yonce (1996). Previous research has suggested that indicator validities of $1.25 S D$ or higher are suitable for taxometric analysis (Meehl, 1995a). The ASP-6 and ASP-4 indicators were both deemed to be highly valid (ASP-6 $M=2.12$, range $=1.70-2.29$; $A S P-4$ $M=2.07$, range $=2.00-2.19$ )

\section{Suitability Test of the ASP Indicators}

Previous research has suggested that for data to be considered suitable for taxometric analysis, the plots from the simulated taxonic and dimensional data generated, using data with similar distributional characteristic to the research data, should be readily distinguishable from one another (Ruscio et al., 2006, 2007). In other words, if taxonic and dimensional data are generated to simulate the research data, and the output plots from the simulations are not differentiable (i.e., both sets look taxonic or both sets appear dimensional), then it is unlikely that the data will be able to provide a clear answer regarding taxonicity. Therefore, as a preliminary test of the suitability of the present research data for conducting a taxometric analysis of anxiety sensitivity, the simulated taxonic and dimensional plots generated based on the ASP-6 and ASP-4 indicators were examined. Visual analysis of the simulated MAXEIG and MAMBAC graphs revealed that the simulated taxonic plots consistently demonstrated peaks, whereas the simulated dimensional plots did not. See Figures 1 and 2 for simulated taxonic and dimensional plots based on the ASP-6 and ASP-4 data respectively. Furthermore, the simulated taxonic plot produced by L-Mode demonstrated a clear bimodal distribution (i.e., two distinct peaks), which was contrasted with the unimodal distribution of the simulated dimensional plot. Based on the easy discrimination between the simulated taxonic and dimensional findings, the ASP-6 and ASP-4 indicators should produce reliable and interpretable plots when submitted to taxometric analysis.

\section{ASP Results}

The two sets of ASP indicators were submitted to MAXEIG analysis first. MAXEIG generated six plots, none of which evidenced a peak that would be consistent with a latent taxon. Rather, all the plots were relatively flat, with minor fluctuations around a mean eigenvalue, thus appearing to indicate a dimensional solution. To reduce the 
quantity of graphical output, only the averaged plot for each taxometric procedure is presented. As can be seen in Figures 1 and 2, when the averaged MAXEIG curves were compared with curves generated using simulated taxonic and dimensional data with similar distributional characteristics, the data plot more closely resembled the simulated dimensional plots. The simulated taxonic plots, on the other hand, revealed distinct peaks that were absent in the averaged research data curves. The Ruscio (2006) programs also provide a comparison curve fit index (CCFI), which is a numerical gauge of whether the data plots are more consistent with a taxon or dimension. The CCFI scores range between 0 and 1 , with lower scores $(<0.5)$ being interpreted as more suggestive of a dimensional solution. Specifically, a score of 0.00 represents the strongest support for a dimension, whereas a score of 1.0 represents the strongest support for a taxon. Recent research has indicated that the CCFI is an effective method of discriminating taxonic and dimensional data (Ruscio, in press; Ruscio et al., 2007; Ruscio \& Marcus, 2007). The CCFIs for the ASP-6 and ASP-4 data were .34 and .31, respectively, which provides further support for a continuous conceptualization of anxiety sensitivity.

As noted above, taxometric procedures rely on replication of findings across independent procedures to ensure accuracy of results (Meehl, 1995a). Thus, even though the MAXEIG procedure supplied evidence for a dimensional structure, three additional taxometric procedures were applied to the ASP data. Despite the aforementioned limitations of the MAXCOV procedure when applied to indicators with skewed distributions, the vast majority of previous anxiety sensitivity taxometric studies have relied on MAXCOV as their primary method of analysis. Thus, to be consistent with the methodology of previous reports, the present research also submitted the ASP data to MAXCOV analyses. The ASP-6 and ASP-4 indicator sets generated a total of 72 MAXCOV plots. Again, none of the plots demonstrated clear peaks that would be consistent with a taxon. Rather, the MAXCOV curves were relatively flat or slightly rising toward the right-plot shapes that are consistent with MAXCOV analyses of nontaxonic data. In addition, as can be seen in Figures 1 and 2, the averaged MAXCOV plots for the ASP-6 and ASP-4 data were comparable to those produced using simulated dimensional data. In contrast, the simulated taxonic plots evidenced distinctive peaks, which were absent from the ASP data plots. The CCFIs for the ASP-6 and ASP-4 data were .36 and .32, respectively. Thus, consistent with the MAXEIG, MAXCOV analyses of the present research data provided additional 
support for a dimensional conceptualization of anxiety sensitivity.

MAMBAC analyses were conducted as a third consistency test. The two sets of ASP indicators generated a total of 42 MAMBAC plots. None of the MAMBAC plots evidenced a distinct peak that would be consistent with a taxon. Rather, all 42 of the plots depicted the typical bowl shape that is typical of a dimensional variable (See Figures 1 and 2 for the averaged curves for the ASP-6 and ASP-4 data, respectively). A comparison of the ASP data plots with the simulated taxonic and dimensional plots revealed that the data curves closely mirrored those of the simulated dimensional plots. The CCFI scores for the ASP-6 and ASP-4 MAMBAC analyses were .33 and .40, which again is suggestive of a dimensional solution.

L-Mode analyses were conducted as a fourth consistency test. The factor score density distributions generated by the ASP-6 and ASP-4 indicators both demonstrated a single peak (see Figures 1 and 2), which is consistent with the typical unimodal distributions produced by dimensional data. In contrast to the ASP data plots and the dimensional simulations, the simulated taxonic plots showed two distinct modal peaks, which are characteristic of taxonic variables. Thus, the L-Mode results are consistent with those of MAXEIG, MAXCOV, and MAMBAC in indicating that anxiety sensitivity, as measured by the two sets of ASP indicators, is nontaxonic at the latent level.

\section{STUDY 2}

The dimensional findings indicated by taxometric analysis of the ASP data contradict the reports of previous anxiety sensitivity taxometric studies, which have consistently indicated taxonic latent structure. The primary methodological difference between previous inquiries and Study 1 (above) was that the present research utilized a unique measure of anxiety sensitivity (the ASP). Thus, one potential explanation for the discrepancy in findings is that measurement issues associated with the ASP (e.g., psychometric properties, question or response format, construct actually being measured, levels of nuisance covariance among ASP indicators, etc.) may not allow for the detection of an anxiety sensitivity taxon. The purpose of Study 2 was to test this hypothesis by conducting a taxometric analysis of anxiety sensitivity using ASI-R data collected from a second large sample. 


\section{Method}

\section{Participants}

Participants consisted of 744 male $(n=206)$ and female $(n=538)$ undergraduate students from a large university who volunteered to participate in this study in exchange for course credit. Participants were able to select from several studies being conducted in the psychology department via an online management system that briefly described the study and time requirements. Participants ranged in age from 18 to $55(M=20.52, S D=4.06)$, and ethnicities included: 52\% White, 44.1\% African American, 0.5\% Hispanic, 0.8\% Asian, 0.3\% Native American, and 1.2\% other.

\section{Measures}

$A S I-R$. The ASI-R (Taylor \& Cox, 1998b) is a selfreport questionnaire that assesses fear of anxiety and anxiety-related sensations. The revised version of the ASI consists of 36 questions (as opposed to 16 on the original $\mathrm{ASI}$ ) and assesses in greater depth the major subcomponents of anxiety sensitivity. Exploratory factor analysis has provided evidence for a hierarchical structure of the ASIR, with a general anxiety sensitivity factor and four lower order factors (Deacon et al., 2003; Taylor \& Cox, 1998b). Construct validity for the ASI-R has been established based on significant correlations with the original version of the ASI ( $r=.94$; Taylor \& Cox, 1998b). The ASI-R has also been shown to display adequate criterion validity, in that patients with anxiety disorder diagnoses tend to score higher than nonpatients (e.g., Deacon \& Abramowitz, 2006). The ASI-R has demonstrated excellent internal consistency $(\mathrm{a}=.95)$, with all 36 items showing adequate item-total correlations $(M=.58$, range $=.40-.71$; Deacon et al., 2003). The ASI-R was selected for use in the present study based on its well-documented psychometric properties and its ability to comprehensively assess the full spectrum of the anxiety sensitivity construct.

\section{Structured Clinical Interview for DSM-I Axis I} Disorders-Anxiety Disorders Module. The Anxiety Disorders Module of the Structured Clinical Interview for DSM-I Axis I Disorders (SCID-I; First, Spitzer, Gibbon, \& Williams, 2001) is a semistructured interview designed to assist clinically trained administrators in making reliable Axis I diagnoses. The purpose of including the structured interview was to determine the rate of anxiety disorders in the sample to ensure that the rates of anxiety sensitivity-related psychopathology among the 
sample were sufficient enough to allow for taxon detection

if in fact an anxiety sensitivity taxon existed.

FIGURE 1

Plots for the Anxiety Sensitivity Profile Data Based on the Six-Factor Solution (left), as well as Simulated Taxonic Plots (center), and Simulated Dimensional Plots (right)
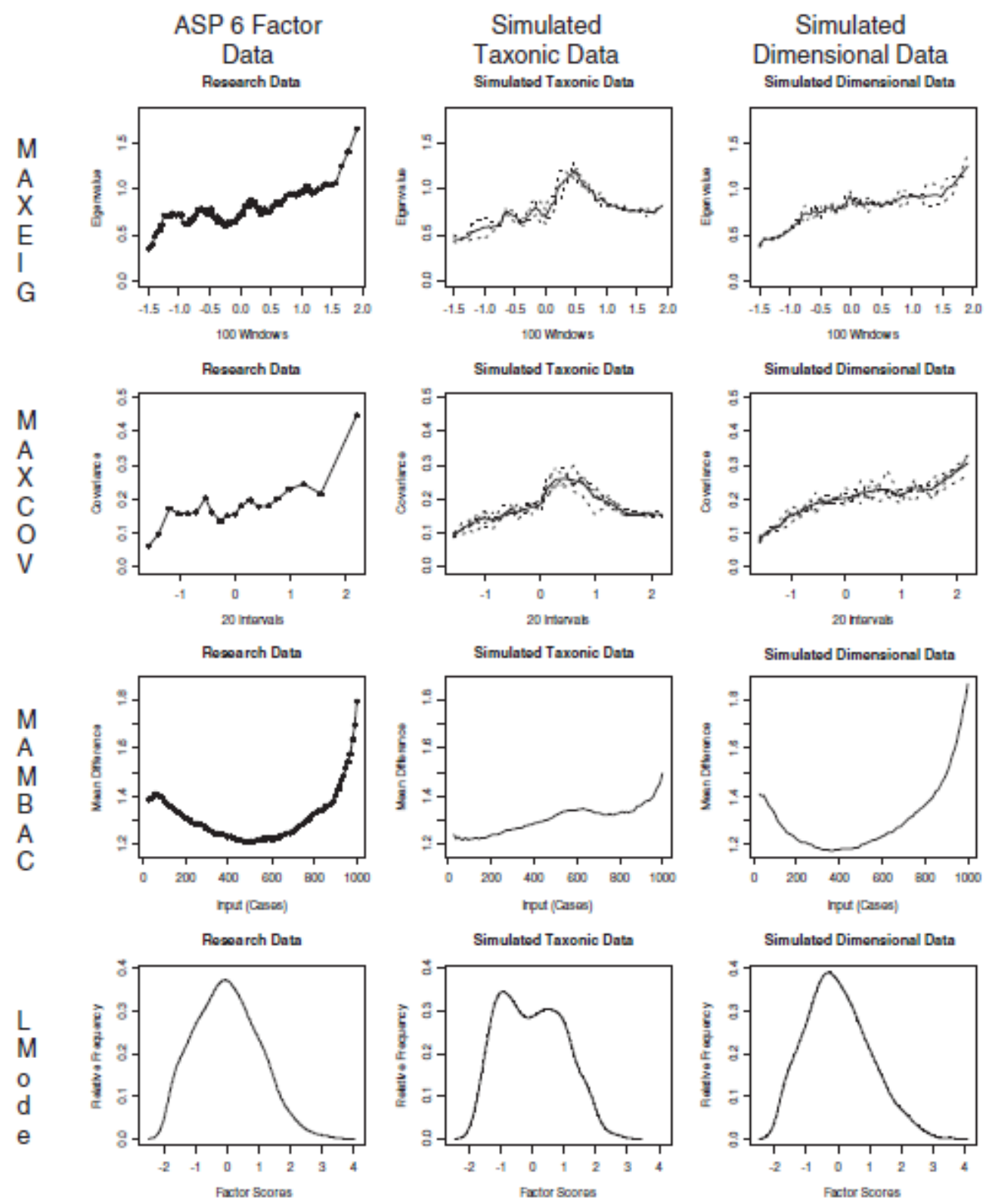

NOIE: Averaged MAXEIG (top), MAXCOV (second row), MAMBAC (third row), and L-Mode (bottom). 
FIGURE 2

Plots for the Anxiety Sensitivity Profile Data Based on the Four-Factor Solution (left), as well as Simulated Taxonic Plots (center), and Simulated Dimensional Plots (right)
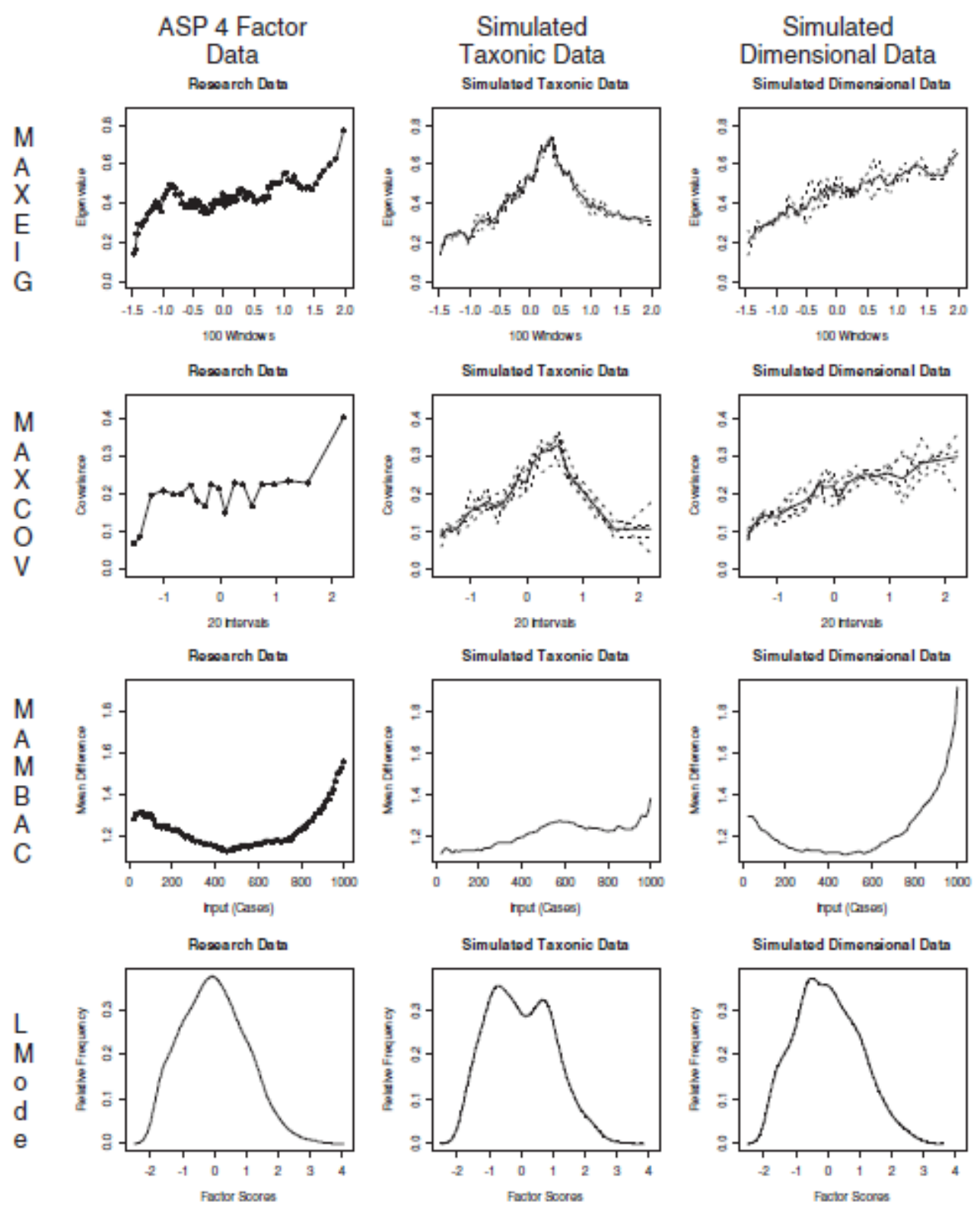

NOIE: Averaged MAXEIG (top), MAXCOV (second row), MAMBAC (third row), and L-Mode (bottom). 


\section{Results}

\section{SCID Results}

In addition to completing a measure of anxiety sensitivity, participants in Study 2 were also administered a structured diagnostic interview to determine the relative rates of anxiety psychopathology in the sample. Results indicated that approximately $22 \%$ of the sample met $D S M-I V$ diagnostic criteria for a current anxiety disorder at the time of the study. Table 1 displays a breakdown of the rates of each anxiety disorder in the Study 2 sample. Thus, the Study 2 sample was known to contain an adequate representation of anxiety sensitivity-related psychopathology and should therefore allow for the detection of an anxiety sensitivity taxon (if one exists).

TABLE 1

Rates of Anxiety Disorder Diagnoses in the Study 2 Sample

\begin{tabular}{lc}
\hline Anxiety Disorder & Study 2 Prevalence Rates (\%) \\
\hline Panic disorder & 2.8 \\
Agoraphobia & 1.5 \\
Specific phobia & 11.8 \\
Social phobia & 4.3 \\
Obsessive compulsive disorder & 2.4 \\
Posttraumatic stress disorder & 5 \\
Generalized anxiety disorder & 3.6 \\
Any anxiety disorder & 22.2 \\
\hline
\end{tabular}

\section{ASI-R Indicator Selection}

As noted above, principal components analysis has indicated that the ASI-R assesses four lower order factors: (a) fear of respiratory symptoms, (b) fear of publicly observable anxiety reactions, (c) fear of cardiovascular symptoms, (d) fear of cognitive dyscontrol (Taylor \& Cox, 1998b). At least one previous taxometric analysis of anxiety sensitivity that indicated a taxonic solution (Bernstein, Zvolensky, Kotov, et al., 2006) created indicators by summing the items that loaded on each of these lower order factors. Thus, consistent with the Bernstein et al. (2006) study, ASI-R items loading on each of the lower order factors were summed to create four composite indicators (Ruscio, Borkovec, \& Ruscio, 2001). 
The validity and nuisance covariance of each ASI-R indicator was examined. Nuisance correlations among the ASI-R indicators were much lower than in the two sets of ASP indicators (average correlation of .13 for the taxon class and .17 for the complement class). In the total sample, the average correlation among the ASI-R indicators was .48. Furthermore, the ASI-R indicators were estimated to have ample validity $(M=1.74$, range $=1.37-2.03)$.

FIGURE 3

Plots for the Anxiety Sensitivity Index-Revised Data, as well as Simulated Taxonic Plots (center), and Simulated Dimensional Plots (right)

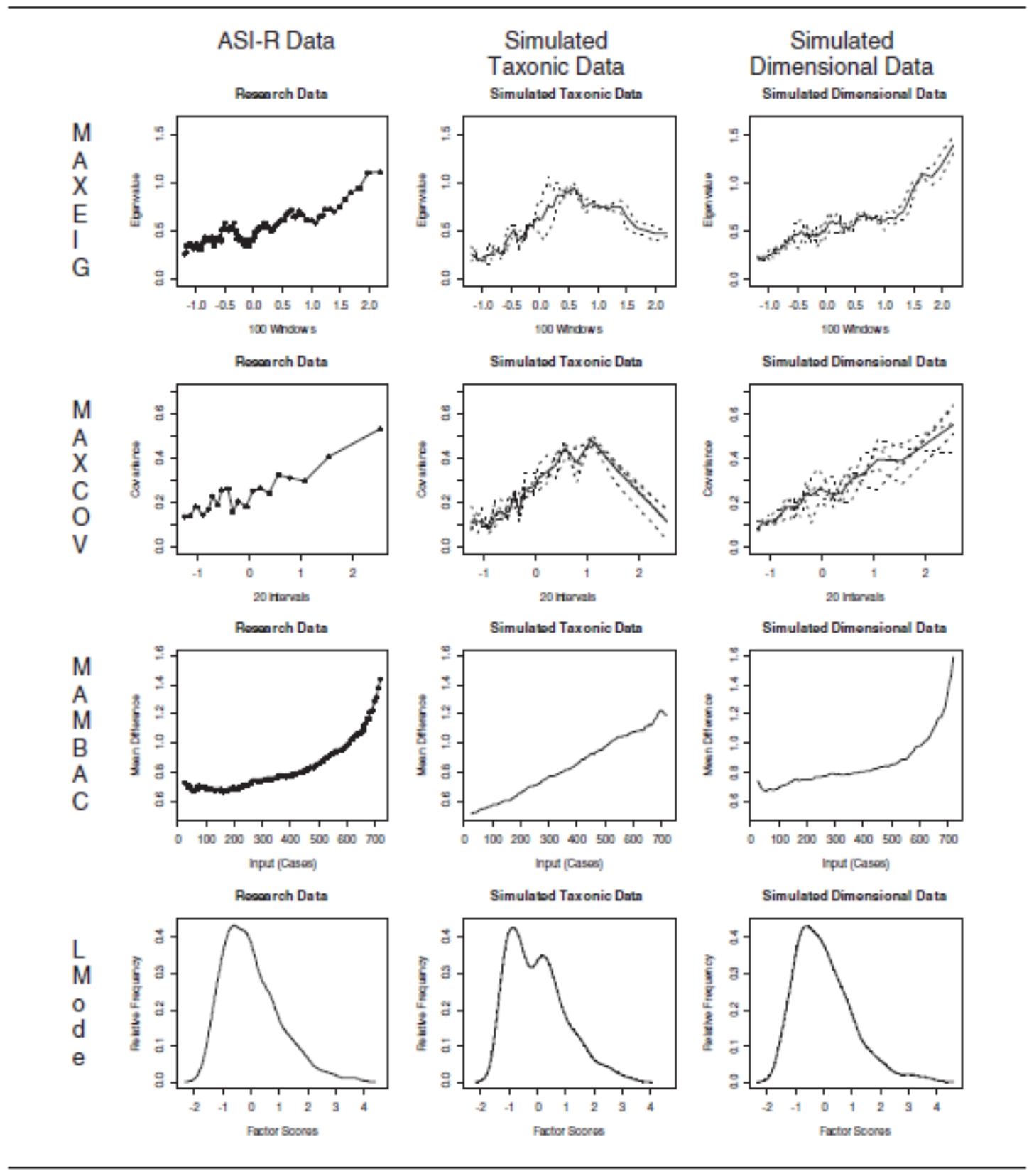

NOTE: Averaged MAXEIG (top), MAXCOV (second row), MAMBAC (third row), and L-Mode (bottom). 


\section{Suitability Test for ASI-R Data}

An initial suitability test of the ASI-R indicators revealed that the simulated taxonic plots generated distinct peaks for the MAXEIG and MAXCOV procedures, whereas the simulated dimensional plots did not. The simulated taxonic plot produced by L-Mode evidenced two distinct peaks, whereas the simulated dimensional plot was clearly unimodal. However, it should be noted that the simulated taxonic MAMBAC plot demonstrated a right side cusp, suggesting that results of the MAMBAC procedure should be interpreted with caution. Refer to Figure 3 for the averaged simulation curves. Based on the low levels of nuisance covariance, high validity, and clear differences in plot shapes of the simulated taxonic and dimensional plots, the ASI-R data were deemed appropriate for taxometric analysis.

TABLE 2

Means, Standard Deviations, and Ranges of the Base-Rate Estimates Generated by the Anxiety Sensitivity Profile-Six Factor Solution, Anxiety Sensitivity Profile-Four Factor Solution, and Anxiety Sensitivity Index-Revised Data Sets

\begin{tabular}{|c|c|c|c|c|c|c|c|}
\hline \multirow[b]{2}{*}{ Indicator } & \multicolumn{2}{|c|}{ MAXEIG } & \multicolumn{2}{|c|}{ MAXCOV } & \multicolumn{2}{|c|}{ MAMBAC } & \multirow[t]{2}{*}{ L-Mode } \\
\hline & M (SD) & Range & $\mathrm{M}$ (SD) & Range & $\mathrm{M}$ (SD) & Range & \\
\hline ASP-6 & $.26(.08)$ & $.16-39$ & $.24(.21)$ & $.05-.77$ & $.44(.06)$ & $.34-60$ & .49 \\
\hline ASP-4 & $.35(.24)$ & $.16-.71$ & $.30(.26)$ & $.09-.81$ & $.46(.06)$ & $.37-.56$ & .49 \\
\hline ASI-R & $.28(.19)$ & $.09-.54$ & $.17(.08)$ & $.08-.31$ & $.34(.12)$ & $.18-.57$ & .56 \\
\hline
\end{tabular}

NOTE: ASP-6 = Anxiety Sensitivity Profile-Six Factor Solution; ASP-4 = Anxiety Sensitivity Profile-Four Factor Solution; ASI-R = Anxiety Sensitivity Index-Revised.

\section{ASI-R Results}

Four MAXEIG plots were produced by the four ASI-R indicators. Refer to Figure 3 for MAXEIG averaged plots based on the ASI-R data, including comparison dimensional and taxonic plots created using simulated data with similar distributional characteristics. Three of the four MAXEIG plots appeared to be relatively flat, with slight variations around a mean eigenvalue and were rated as dimensional. The remaining plot appeared to rise toward the right side, though no noticeable peak was present. When the ASI-R data-generated plots were compared to curves produced by simulated dimensional and taxonic data, the ASI-R plots were found to be much more similar to the simulated dimensional plots. Furthermore, the CCFI score was .30, which is also consistent with a latent dimension. 
The ASI-R data were also submitted to MAXCOV analyses, producing a total of 12 MAXCOV curves. Again, none of the 12 plots produced a clear peak that would be indicative of a taxon. Furthermore, as can be seen in Figure 3, the averaged ASI-R data curve closely resembled the simulated dimensional curve. In contrast, the simulated taxonic plot evidenced a distinctive peak. Analysis of the CCFI score (.28) provided further convergent support for the absence of a latent taxon.

MAMBAC analyses were conducted next, producing a total of 12 data plots. Eleven of the plots were relatively flat or dish shaped, with a slight rise toward the right, which is consistent with a dimensional variable that has been assessed using indicators with skewed distributions (Ruscio \& Ruscio, 2002; Ruscio et al., 2004). The remaining plot demonstrated a potential cusp on the right rise and thus appeared to be ambiguous. The data plots closely resembled those produced by simulated dimensional data with similar degrees of skew (see Figure 3). The CCFI score was .31, which is suggestive of the absence of a taxon. However, because of the apparent lack of a clear peak in the simulated taxonic MAMBAC plot, caution should be exercised in interpreting the results of the MAMBAC analyses of the ASI-R data.

The L-Mode factor analysis plot for the general anxiety sensitivity factor depicted a single peak (see Figure 3), which complements the MAXEIG and MAMBAC results and supports a dimensional structure for anxiety sensitivity. In contrast to the ASI-R data plot and the simulated dimensional plot, the simulated taxonic plot demonstrated a bimodal distribution.

\section{Analysis of Base Rate Estimates}

In addition to generating plots that can be visually examined for evidence of taxonicity or dimensionality, MAXEIG, MAXCOV, MAMBAC, and L-Mode factor analyses also produce a series of base rate estimates for the proposed taxon (if one exists). Previous taxometric studies of anxiety sensitivity have reported relatively consistent base rate estimates, typically ranging between $12 \%$ and $20 \%$. However, an examination of the base rate estimates associated with the ASP-6, ASP-4, and ASI-R data sets revealed significantly higher and more variable estimates than had been reported in previous studies, with mean estimates ranging from .17 to .56 (see Table 2). Thus, an analysis of base rate estimates also failed to replicate the relatively consistent base rate estimates reported in previous 
anxiety sensitivity taxometric studies. Furthermore, Ruscio et al. (2006) have convincingly argued that consistency of base rate estimates is an unreliable indicator of taxonicity, because skewed dimensional data can produce estimates that are highly consistent, and taxonic data can produce estimates that vary, especially when comparing base rate estimates across the independent taxometric procedures. In other words, although it is reasonable to expect some consistency in the base rate estimates produced by taxonic data, such consistency is an unreliable indicator for taxonicity, being especially vulnerable to false positive errors.

\section{Summary of Taxometric Analyses}

In summary, four taxometric procedures-MAXEIG, MAXCOV, MAMBAC, and L-Mode-were used to analyze the latent structure of anxiety sensitivity in two large, nonclinical samples, as measured by the ASP and ASI-R. Multiple indicator sets representing the anxiety sensitivity construct produced more than 150 data plots. Overall, results of the present study suggest that in these samples, and as measured by the ASP and ASI-R, anxiety sensitivity is nontaxonic or dimensional at the latent level.

\section{GENERAL DISCUSSION}

The vast majority of anxiety sensitivity research conducted over the past 25 years has operated on the assumption that anxiety sensitivity is a dimensional variable, with individuals differing by degree of anxiety sensitivity experienced, rather than by type of anxiety sensitivity. Although an initial attempt by Taylor and colleagues (1999) to evaluate the latent structure of anxiety sensitivity appeared to be supportive of the dimensional solution, recent taxometric research has raised questions regarding the accuracy of the dimensional assumption. Specifically, several taxometric analyses have been published indicating that anxiety sensitivity is more accurately conceptualized as a categorical variable, with some individuals experiencing a pathological form of anxiety sensitivity. The purpose of the present research was to investigate the latent structure of anxiety sensitivity using a comprehensive series of taxometric procedures, two large, nonclinical samples, and a unique measure of anxiety sensitivity (the ASP), as well as a commonly used extension of the ASI (the ASI-R). Contrary to the study hypotheses and previous reports indicating that anxiety sensitivity is taxonic at the latent 
level, the results of four mathematically independent taxometric procedures-MAXEIG, MAXCOV, MAMBAC, and L-Mode-applied to data from two large nonoverlapping samples were interpreted by four experienced taxometricians as providing consistent and convergent evidence for a dimensional conceptualization of anxiety sensitivity. In fact, the more than 150 anxiety sensitivity data plots generated by the various taxometric procedures, not a single plot evidenced a shape that would be interpreted as clearly indicative of a taxon. Furthermore, an inspection of the base rate estimates revealed substantial variability across the procedures and the data sets, with individual plot estimates ranging from $9 \%$ to $81 \%$, and therefore failing to cohere around the base rates (11-18\%) noted in previous anxiety sensitivity taxometric studies using samples with similar demographic characteristics.

The dimensional results of the present research are clearly inconsistent with the taxonic reports of Bernstein and colleagues. Initially, it was suspected that the use of a unique anxiety sensitivity measure (the ASP) in Study 1 could be the source of the difference between these dimensional and previous taxonic findings. For example, the psychometric properties or overall structure of the ASP or ASP indicators may have prevented our ability to uncover a taxon that was in fact present. In an effort to evaluate this hypothesis, Study 2 attempted to replicate the findings of previous taxonic reports using the same measure (the ASI-R) and the same indicators as those used by Bernstein, Zvolensky, Kotov, et al. (2006). Despite the similarities in study methods, Study 2 did not reveal any evidence of a latent taxon, thus ruling out measurement differences as a source of divergence. However, the discrepancies in the latent structure of anxiety sensitivity may be partially attributable to the unstable factor structure of most commonly used anxiety sensitivity measures, including the ASI, ASI-R, and ASP (e.g., Deacon et al., 2003; Zvolensky et al., 2003). Researchers have recently developed the ASI3 to overcome some of the psychometric limitations of its predecessors, and initial findings appear promising (Taylor et al., 2007). Thus, further taxometric research using the ASI-3 may be instrumental in enabling researchers to make more definitive inferences regarding the latent structure of anxiety sensitivity.

A second potential explanation for the discrepancy between these findings and those of previous reports concerns the samples utilized in the various studies. Whereas the present research was conducted on two nonclinical samples of American college students, Bernstein and colleagues have reported finding anxiety sensitivity taxonicity 
with fairly consistent base rates across multiple, diverse samples of nonclinical children and adults across several cultures. Thus, it is possible that anxiety sensitivity is categorical in some unique subgroups of the general population and dimensional in others. However, unless the distinction between taxon and complement groups disappears between childhood and young adulthood only in the United States population, it does not appear to be the most parsimonious explanation for the discrepant findings. A third possible explanation for these discrepancies lies in the taxometric procedures themselves. Specifically, the taxometric analyses conducted by Bernstein and colleagues

(2005) and Schmidt et al. (2005) have typically relied heavily on MAXCOV as their primary method of analysis. The MAXCOV procedure is known to possess several important limitations that are directly relevant to the study of anxiety sensitivity latent structure. For example, MAXCOV is performed using nonoverlapping windows, which constricts the number of data points on the resulting output plots. The use of nonoverlapping windows also produces subsamples of unequal size, with fewer cases in the outer intervals than those closer to the center. With skewed indicators, the intervals nearer the longer end of the distribution will contain even fewer cases, thereby increasing the amount of sampling error along that portion of the distribution. As a result, the application of MAXCOV to skewed indicators of a dimensional variable will often produce plots that closely resemble curves generated by a low base-rate taxon (Ruscio \& Ruscio, 2002). In general, anxiety sensitivity scores tend to be positively skewed, a characteristic that was also noted in the present research data indicator distributions $(M=0.44,0.49$, and 1.11 across ASP-6, ASP-4, and ASI-R data, respectively). Thus, to address potential concerns about the limitations of MAXCOV, the present research implemented the MAXEIG procedure. MAXEIG is a multivariate version of the MAXCOV procedure that uses overlapping windows and holds constant the number of cases (and thus the amount of sampling error) in each window. MAXEIG also creates a far greater number of response points, which enhances the interpretability of the resulting data plots (Ruscio et al., 2004).

Results of the MAXEIG procedure strongly supported a dimensional, rather than categorical, conceptualization of anxiety sensitivity. In addition, three consistency tests (including MAXCOV) confirmed these findings by failing to uncover any evidence of a latent taxon. Therefore, it is possible that previous taxonic findings of anxiety sensitivity may be at least partially attributable to selection of taxometric procedures. Although several potential explanations for these discrepancies have been suggested, the 
exact source of the discrepant findings of taxometric analyses of anxiety sensitivity remains unclear. Further research will be required to investigate these and other alternative explanations to determine the exact source of the divergent findings.

The results of the present research have several important implications for our understanding of fears associated with anxiety-related sensations. First, the general assumption running throughout the anxiety sensitivity literature that anxiety sensitivity is continuous or dimensional appears to be correct in at least some populations. A second important theoretical implication relates to the search for the etiology of anxiety sensitivity. Although the origins of anxiety sensitivity remain unclear, and the present study is not intended to directly address such issues, knowing the latent structure of a variable can help provide researchers with some direction for etiological investigation. Most important, a dimensional solution indicates that researchers should not be focused on trying to uncover a specific all-ornone genetic factor, a single environmental variable, or a specific combination of these factors that predict the type of anxiety sensitivity an individual will experience. Rather, latent dimensional structures are more consistent with graded and additive etiological models (Haslam, 1997). Accordingly, anxiety sensitivity researchers should focus their efforts on investigating the effects of various combinations or interactions of specific life events and hereditary factors on the development of higher levels of anxiety sensitivity. For example, it may be that high arousal reactivity combined with exposure to stressors in high frequency, severity, or both, contribute to the development of anxiety sensitivity. Additional factors to consider may include whether the individual has received misinformation about the harmfulness of certain sensations or has witnessed others model such fears or parental reinforcement of sick-role behavior related to somatic symptoms in general. The present findings also have important implications for the assessment of anxiety sensitivity. Some investigators have elected to dichotomize the anxiety sensitivity construct for research purposes (e.g., Asmundson et al., 1994; Donnell \& McNally, 1990), thus creating unnatural breaks in the data. Although dichotomization of a continuous variable can be desirable or necessary for feasibility issues, artificially dichotomizing (e.g., high vs. low anxiety sensitivity) a construct that is truly dimensional can also lead to an unnecessary loss of information (Cohen, 1983). Furthermore, any cut-points on anxiety sensitivity measures used to select or exclude participants from research do not correspond to natural divisions in the data and may distort findings. Therefore, anxiety sensitivity 
researchers are encouraged to assess the entire continuum of anxiety sensitivity, when possible, and to use statistical procedures, such as multiple regression analysis, that do not necessitate artificial dichotomization.

In sum, a series of recently published taxometric investigations of anxiety sensitivity have suggested that the latent structure of anxiety sensitivity may be categorical at the latent level. However, the present research was unable to replicate these findings. Specifically, results from four mathematically independent taxometric procedures (MAXEIG, MAXCOV, MAMBAC, and L-Mode), applied to data from two large nonclinical samples using two distinct measures of anxiety sensitivity, provided consistent and convergent evidence for a dimensional conceptualization of anxiety sensitivity. Although several potential explanations for the discrepancy between the findings of the present research and those of previous reports are discussed, additional research will be required to determine the exact source of the incongruity.

\section{NOTES}

1. Taxometric analyses were also conducted using programs devised by Waller and Meehl (1998), Meehl and Yonce $(1994,1996)$, and Grove (2007). Results of those analyses were consistent with those produced by the Ruscio programs in supporting a dimensional latent structure. Thus, for the sake of brevity, only the analyses conducted using the Ruscio programs are detailed here. However, all output is available on request.

2. A detailed description of how the Ruscio taxometric programs estimate taxon and complement base rates, even among apparently dimensional variables, can be found in Ruscio (2006).

3. As noted in the Method section, four experienced taxometricians (i.e., individuals who had previously authored one or more published taxometric articles) interpreted the plot shapes of MAXEIG, MAXCOV, MAMBAC, and L-Mode output. It should be noted that all four taxometricians were in agreement that the ASP and ASI-R data plots were indicative of a dimension. Taxometricians included David Marcus, Bradley Green, Randolph Arnau, and Joshua Broman-Fulks.

\section{REFERENCES}

Arnau, R. C., Thompson, R. L., \& Cook, C. (2001). Do different response formats change the latent structure of responses? An empirical investigation using taxometric analyses. Educational and Psychological Measurement, 61, 23-44. 
Asmundson, G. J. G., Norton, G. R., Wilson, K. G., \& Sandler, L. S. (1994). Subjective symptoms and cardiac reactivity to brief hyperventilation in individuals with high anxiety sensitivity. Behaviour Research and Therapy, 32, 237-241.

Beach, S. R. H., Amir, N., \& Bau, J. J. (2005). Can sample-specific simulations help detect low base-rate taxonicity? Psychological Assessment, 17, 446-461.

Bernstein, A., Zvolensky, M. J., Kotov, R., Arrindell, W. A., Taylor, S., Sandin, B., et al. (2006). Taxonicity of anxiety sensitivity: A multinational analysis. Journal of Anxiety Disorders, 20, 1-20.

Bernstein, A., Zvolensky, M. J., Norton, P. J., Schmidt, N. B., Taylor, S., Forsyth, J. P., et al. (2007). Taxometric and factor analytic models of anxiety sensitivity: Integrating approaches to latent structural research. Psychological Assessment, 19, 74-87.

Bernstein, A., Zvolensky, M. J., Stewart, S. H., Comeau, M. N., \& LeenFeldner, E. W. (2006). Anxiety sensitivity taxonicity across gender among youth. Behaviour Research and Therapy, 44, 679-698.

Bernstein, A., Zvolensky, M. J.,Weems, C., Stickle, T., \& Leen-Feldner, E. W. (2005). Taxonicity of anxiety sensitivity: An empirical test among youth. Behaviour Research and Therapy, 43, 1131-1155.

Broman-Fulks, J. J., Berman, M. E., Rabian, B., \& Webster,M. J. (2004). Effects of aerobic exercise on anxiety sensitivity. Behaviour Research and Therapy, 42, 125-136.

Broman-Fulks, J. J., Ruggiero, K. J., Kilpatrick, D. G., Resnick, H. S., Green, B. A., \& Saunders, B. E. (2006). Taxometric investigation of posttraumatic stress disorder: Data from two nationally representative samples. Behavior Therapy, 37, 364-380.

Chambless, D. L., Caputo, G. C., Bright, P., \& Gallagher, R. (1984). Assessment of fear of fear in agoraphobics: The Body Sensations Questionnaire and the Agoraphobic Cognitions Questionnaire. Journal of Consulting and Clinical Psychology, 52, 1090-1097.

Cohen, J. (1983). The cost of dichotomization. Applied Psychological Measurement, 7, 249-253.

Deacon, B. J., \& Abramowitz, J. S. (2006). Anxiety sensitivity and its dimensions across the anxiety disorders. Journal of Anxiety Disorders, 20, 837-857.

Deacon, B. J., Abramowitz, J. S., Woods, C. M., \& Tolin, D. F. (2003). The Anxiety Sensitivity Index-Revised: Psychometric properties and factor structure in two nonclinical samples. Behaviour Research and Therapy, 41, 1427-1449. 
Donnell, C. D., \& McNally, R. J. (1990). Anxiety sensitivity and panic attacks in a nonclinical population. Behaviour Research and Therapy, $28,83-85$.

Ehlers, A. (1995). A one-year prospective study of panic attacks: Clinical course and factors associated with maintenance. Journal of Abnormal Psychology, 104, 164-172.

First, M. B., Spitzer, R. L., Gibbon, M., \& Williams, J. B. W. (2001). Structured Clinical Interview for DSM-IV-TR Axis I Disorders, Research Version, Non-patient Edition. (SCID-I/NP). New York: Biometrics Research, New York State Psychiatric Institute.

Grove, W. M. (2007). William M. Grove's downloadable programs/ source code. Retrieved June 17, 2007, from http://www.psych.umn .edu/faculty/grove/computer-code.htm

Harrington, P. J., Telch, M. J., Abplanalp, B., \& Hamilton, A. C. (1995, November). Lowering anxiety sensitivity in nonclinical subjects:

Preliminary evidence for a panic prevention program. Paper presented at the 29th annual meeting of the Association for the Advancement of Behavior Therapy, Washington, DC.

Haslam, N. (1997). Evidence that male sexual orientation is a matter of degree. Journal of Personality and Social Psychology, 73, 862-870.

Lenzenweger, M. F., \& Korfine, L. (1992). Confirming the latent structure and base rate of schizotypy: A taxometric analysis. Journal of Abnormal Psychology, 101, 567-571.

Maller, R. G., \& Reiss, S. (1992). Anxiety sensitivity in 1984 and panic attacks in 1987. Journal of Anxiety Disorders, 6, 241-247.

McNally, R. J. (2002). Anxiety sensitivity and panic disorder. Biological Psychiatry, 52, 938-946.

Meehl, P. E. (1995a). Bootstraps taxometrics: Solving the classification problem in psychopathology. American Psychologist, 50, 266-275.

Meehl, P. E. (1995b). Extension of the MAXCOV-HITMAX taxometric procedure to situations of sizable nuisance covariance. In D. Lubinski \& R. V. Davis (Eds.), Assessing individual differences in human behavior (pp. 81-92). Palo Alto, CA: Davies-Black.

Meehl, P. E., \& Golden, R. R. (1982). Taxometric methods. In J. N. Butcher \& P. C. Kendall (Eds.), The handbook of research methods in clinical psychology (pp. 127-181). New York:Wiley. 
Meehl, P. E., \& Yonce, L. J. (1994). Taxometric analysis I: Detecting taxonicity with two quantitative indicators using means above and below a sliding cut (MAMBAC procedure). Psychological Reports, 74, 1059-1274.

Meehl, P. E., \& Yonce, L. J. (1996). Taxometric analysis II: Detecting taxonicity using covariance of two quantitative indicators in successive intervals of a third indicator (MAXCOV procedure). Psychological Reports, 78, 1091-1227.

Olatunji, B. O., Sawchuck, C. N., Deacon, B. J., Tolin, D. F., Lilienfeld, S. O.,Williams, N. L., et al. (2005). The Anxiety Sensitivity Profile revisited: Factor structure and psychometric properties in two nonclinical samples. Journal of Anxiety Disorders, 19, 603-625.

Peterson, R. A., \& Reiss, S. (1992). Anxiety Sensitivity Index Manual (2nd ed.). Worthington, $\mathrm{OH}$ : International Diagnostic Systems.

R Development Core Team. (2005). R: A language and environment for statistical computing. R Foundation for Statistical Computing, Vienna, Austria (ISBN 3-900051-07-0). Available from the R Project for Statistical Computing Web site, http://www.R-project.org

Reiss, S., \& McNally, R. J. (1985). The expectancy model of fear. In S. Reiss \& R. R. Bootzin (Eds.), Theoretical issues in behavior therapy. London: Academic Press.

Ruscio, A. M., Borkovec, T. D., \& Ruscio, J. (2001). A taxometric investigation of the latent structure of worry. Journal of Abnormal Psychology, 110, 413-422.

Ruscio, A. M., \& Ruscio, J. (2002). The latent structure of analogue depression: Should the Beck Depression Inventory be used to classify groups? Psychological Assessment, 14, 135-145.

Ruscio, A. M., Ruscio, J., \& Keane, T. M. (2002). The latent structure of posttraumatic stress disorder: A taxometric investigation of reactions to extreme stress. Journal of Abnormal Psychology, 111, 290-301.

Ruscio, J. (2006). Documentation of program code to perform taxometric analyses and simulate comparison data in the $\mathrm{R}$ language.

Available from http://www.taxometricmethod.com

Ruscio, J., Ruscio, A. M., \& Keane, T. M. (2004). Using taxometric analysis to distinguish a small latent taxon from a latent dimension with positively skewed indicators: The case of Involuntary Defeat Syndrome. Journal of Abnormal Psychology, 113, 99-108.

Ruscio, J., Ruscio, A. M., \& Meron, M. (2007). Applying the bootstrap to taxometric analysis: Generating empirical sampling distributions to help interpret results. Multivariate Behavioral Research, 44, 349-386. 
Ruscio, J. (in press). Taxometric analysis: An empirically grounded approach to implementing the method. Criminal Justice and Behavior.

Ruscio, J., Haslam, N., \& Ruscio, A. M. (2006). Introduction to the taxometric method: A practical guide. Mahwah, NJ: Lawrence Erlbaum.

Ruscio, J., \& Marcus, D. K. (2007). Detecting small taxa using simulated comparison data: A reanalysis of Beach, Amir, and Bau's (2005) data. Psychological Assessment, 19, 241-246.

Ruscio, J., \& Ruscio, A. M. (2000). Informing the continuity controversy: A taxometric analysis of depression. Journal of Abnormal Psychology, 109, 473-487.

Schmidt, N. B., Kotov, R., Lerew, D. R., Joiner, T. E., \& Ialongo, N. S. (2005). Evaluating latent discontinuity in cognitive vulnerability to panic: A taxometric investigation. Cognitive Therapy and Research, $29,673-690$.

Schmidt, N. B., Lerew, D. R., \& Jackson, R. J. (1997). The role of anxiety sensitivity in the pathogenesis of panic: Prospective evaluation of spontaneous panic attacks during acute stress. Journal of Abnormal Psychology, 106, 355-364.

Schmidt, N. B., Lerew, D. R., \& Trakowski, J. J. (1997). Body vigilance in panic disorder: Evaluating attention to bodily perturbations. Journal of Consulting and Clinical Psychology, 65, 214-220.

Schmidt, N. B., Zvolensky, M. J., \& Maner, J. K. (2006). Anxiety sensitivity: Prospective prediction of panic attacks and Axis I pathology. Journal of Psychiatric Research, 40, 691-699.

Silverman,W. K., Fleisig,W., Rabian, B., \& Peterson, R. A. (1991). Child Anxiety Sensitivity Index. Journal of Clinical Child Psychology, 20, 162-168.

Taylor, S., \& Cox, B. J. (1998a). Anxiety sensitivity: Multiple dimensions and hierarchic structure. Behaviour Research and Therapy, $36,37-57$.

Taylor, S., \& Cox, B. J. (1998b). An expanded Anxiety Sensitivity Index: Evidence for a hierarchic structure in a clinical sample. Journal of Anxiety Disorders, 12, 463-483.

Taylor, S., Rabian, B., \& Federoff, I. C. (1999). Anxiety sensitivity: Problems, prospects, and challenges. In S. Taylor (Ed.), Anxiety sensitivity: Theory, research, and treatment of the fear of anxiety (pp. 339-353). Mahwah, NJ: Lawrence Erlbaum. 
Taylor, S., Zvolensky, M. J., Cox, B. J., Deacon, B., Heimberg, R. G., Ledley, D. R., et al. (2007). Robust Dimensions of Anxiety Sensitivity: Development and Initial Validation of the Anxiety Sensitivity Index-3. Psychological Assessment, 19, 176-188.

Van der Does, W., Duijsens, I., Eurelings-Bontekoe, E., Verschuur, M., \& Spinhoven, P. (2003). Sensitivity Profile: Dimensional structure and relationship with temperament and character. Psychotherapy and Psychosomatics, 72, 217-222.

Waller, N. G., \& Meehl, P. E. (1998). Multivariate taxometric procedures: Distinguishing types from continua. Thousands Oaks, CA:

Sage.

Watt, M., Stewart, S., Birch, C., \& Bernier, D. (2006). Brief CBT for high anxiety sensitivity decreases drinking problems, relief alcohol outcome expectancies, and conformity drinking motives: Evidence from a randomized controlled trial. Journal of Mental Health, 15, 683-695.

Zvolensky, M. J., Arrindell, W. A., Taylor, S., Bouvard, M., Cox, B. J., Stewart, S. H., et al. (2003). Anxiety sensitivity in six countries.

Behaviour Research and Therapy, 41, 841-859. 